\title{
Análise da abordagem fisioterapêutica no tratamento da disfunção temporomandibular: revisão integrativa
}

\author{
Analysis of the physiotherapy approach in the treatment of temporomandibular \\ dysfunction: integrative review
}

Análisis del enfoque fisioterapéutico en el tratamiento de la disfunción temporomandibular: revisión integrativa

Henessy Bittencourt e Silva ${ }^{1 *}$, Jainy Lima Soares²

\begin{abstract}
RESUMO
Objetivo: Analisar a abordagem fisioterapêutica no tratamento da disfunção temporomandibular, descrevendo os objetivos e resultados das técnicas e recursos utilizados. Métodos: Este estudo trata-se de uma revisão integrativa de literatura realizada a partir da inserção de descritores específicos nas bases de dados PubMed, SciELO e LILACS, onde foram incluídos estudos publicados na íntegra, entre 2010 a 2018, na língua portuguesa e inglesa, e foram excluídos resumos, textos incompletos, periódicos pagos, relatos e estudos de caso, revisões e trabalhos sem fundamentação científica Resultados: A partir da inserção de descritores encontrou-se 169 estudos, os quais, após a filtragem por ano, idioma, gratuidade e texto completo disponível findaram-se em apenas 56. Estes, tiveram seus títulos, resumos e metodologia avaliados, resultando em 7 ensaios clínicos, com amostras entre 10 e 60 pacientes, que utilizaram recursos e técnicas fisioterapêuticas para o tratamento da DTM. Conclusão: Através dos resultados deste estudo, pode-se concluir que a fisioterapia através da eletroterapia, termoterapia e cinesioterapia são eficazes na redução da dor, na mobilidade articular e na melhora da sensibilidade, dessa maneira, contribuindo significativamente para o tratamento da DTM.
\end{abstract}

Palavras-chave: Articulação temporomandibular, Fisioterapia, Modalidades de Fisioterapia.

\begin{abstract}
Introduction: The term temporomandibular dysfunction (TMD) is used to define a set of disorders involving the masticatory muscles, temporomandibular joint and adjacent segments. Its main symptoms are pain, chewing difficulty and constant headache, where, as a treatment, physiotherapy is widely used with the objective of promoting analgesia and mobility of this joint. Objective: To analyze the physiotherapeutic approach in the treatment of temporomandibular dysfunction, describing the objectives and results of the techniques and resources used. Methodology: This study is an integrative review of literature based on the inclusion of specific descriptors in the PubMed, SciELO and LILACS databases, which included studies published in full between 2010 and 2018 in Portuguese and English, and abstracts, incomplete texts, periodicals paid, reports and case studies, revisions and works without scientific basis were excluded. Results: From the insertion of descriptors 169 studies were found, which, after filtering per year, language, gratuity and
\end{abstract}

${ }^{1}$ Pós-Graduando em Osteopatia, Faculdade Integral Diferencial (FACID), Fisioterapeuta (FACID), Caxias-MA. *E-mail: drhenessy89@gmail.com

2Pós-Graduada em Neurorreabilitação Intensiva (UNIFSA), Pós-Graduada em Fisioterapia Intensiva (ICF), Fisioterapeuta (UNIFACEMA), Caxias-MA. 
the available full text was only 56. These had their titles, abstracts and methodology evaluated, resulting in 7 clinical trials, with samples between 10 and 60 patients, that used resources and physiotherapeutic techniques for the treatment of TMD. Conclusion: Through the results of this study, it can be concluded that physical therapy through electrotherapy, thermotherapy and kinesiotherapy are effective in reducing pain, joint mobility and improving sensitivity, thus contributing significantly to the treatment of TMD.

Key-words: Temporomandibular Joint, Physycal Therapy Specialty, Physical Therapy Modalities.

\section{RESUMEN}

Introducción: El término disfunción temporomandibular (DTM) se utiliza para definir un conjunto de disturbios que involucran los músculos masticatorios, la articulación temporomandibular y los segmentos adyacentes. Sus principales síntomas son el dolor, dificultad de masticación y cefalea constante, donde, como tratamiento, la fisioterapia es ampliamente utilizada con el objetivo de promover la analgesia y la movilidad de esa articulación. Objetivo: Analizar el enfoque fisioterapéutico en el tratamiento de la disfunción temporomandibular, describiendo los objetivos y resultados de las técnicas y recursos utilizados. Metodología: Este estudio trata de una revisión integradora de la literatura llevada a cabo a partir de la inserción de los descriptores específicos en PubMed, SciELO y LILACS donde los estudios publicados en su totalidad comprendido entre 2010 a 2018 en el Español y el Inglés, y se excluyeron resúmenes, textos incompletos, periódicos pagados, relatos y estudios de caso, revisiones y trabajos sin fundamentación científica. Resultados: A partir de la inserción de descriptores se encontraron 169 estudios, los cuales, después del filtrado por año, idioma, gratuidad y el texto completo disponible se concluyó en sólo 56 . Estos, tuvieron sus títulos, resúmenes y metodología evaluados, resultando en 7 ensayos clínicos, con muestras entre 10 y 60 pacientes, que utilizaron recursos y técnicas fisioterapéuticas para el tratamiento de la DTM. Conclusión: A través de los resultados de este estudio, se puede concluir que la fisioterapia a través de la electroterapia, termoterapia y cinesioterapia son eficaces en la reducción del dolor, en la movilidad articular y en la mejora de la sensibilidad, de esta manera, contribuyendo significativamente al tratamiento de la DTM.

Palabras-clave: Articulación temporomandibular, Fisioterapia, Modalidades de Fisioterapia.

\section{INTRODUÇÃO}

A articulação temporomandibular (ATM) é considerada a mais complexa do corpo humano, sendo esta capaz de realizar movimentos rotacionais e translacionais devido à articulação dupla do côndilo do osso temporal. A ATM apresenta duas articulações (côndilos), conectados à mandíbula, fator que exige que trabalhem de forma sincronizada entre a oclusão dental, o equilíbrio neuromuscular e a própria articulação. Dessa forma, essa articulação fica vulnerável a alterações funcionais ou patológicas, propiciando desarranjos como a disfunção temporomandibular (DTM) (WEBER et al., 2013).

Bonotto et al. (2014) define a DTM como um conjunto de distúrbios que envolvem os músculos mastigatórios, a ATM e os segmentos adjacentes, sendo esta uma patologia relativamente comum do sistema estomatognático, que afeta cerca de 7 a 15\% da população adulta. Esses distúrbios impactam o equilíbrio dinâmico das estruturas, levando a uma série de sinais e sintomas típicos dessa disfunção, como as dores na face, ATM e/ou músculos mastigatórios, cefaleia, zumbido e vertigem. Quanto aos sinais, é comum ser achado na avaliação do paciente a sensibilidade muscular e da ATM à palpação, limitação e/ou distúrbios do movimento mandibular e ruídos articulares.

O surgimento e a perpetuação das DTM estão condicionados à interação de fatores como trauma, lassidão ligamentar, hábitos parafuncionais, estresse, alterações sistêmicas e mudanças internas e externas na estrutura da articulação temporomandibular, sendo a DTM considerada a causa mais comum de dor crônica da região orofacial, onde, dentre os indivíduos afetados, somente $12 \%$ possuem sintomas graves o suficiente para procurar tratamento (AZATO et al., 2013). 
Para o diagnóstico da disfunção temporomandibular, Milanesi et al. (2013) aponta que diversos questionários, índices e critérios diagnósticos têm sido utilizados, onde, o critério diagnóstico em pesquisa para DTM (RDC/TMD) é apontado como uma das mais bem estruturadas ferramentas para o diagnóstico da DTM, podendo ser aliado ao Índice Anamnésico de Fonseca, por este ser o instrumento mais utilizado para avaliar a severidade da DTM. Ainda, podem ser utilizados exames de imagem que ajudem a complementar o diagnóstico, onde as imagens 3D, gerada por tomógrafos, proporcionam a realização de vários cortes na imagem captada, além de permitir a visão por ângulos diversos.

Em relação ao tratamento, existem diversos técnicas, recursos e protocolos que podem ser utilizados, onde o protocolo primário prioriza as medidas mais simples, reversíveis e menos invasivas, com o foco de controlar e atuar perante a presença de disfunções intracapsulares resultantes de disfunções das superfícies articulares, isto é, de alterações estruturais presentes, onde, o tratamento conservador mostra-se algumas vezes ineficaz. Nesse âmbito, é válido mencionar que existem diversas formas de tratamento para as disfunções internas da ATM que possuem comprovação científica na literatura vigente, como o uso de antiinflamatório não esteroides, o repouso funcional, uso de dispositivos interoclusais, realização de exercícios fisioterápicos de suporte, utilização de injeções intra-articulares de corticosteroide, e, em casos mais graves, a artrocentese, artroscopia e a cirurgia aberta da ATM (BONOTTO et al., 2011).

Por ser uma patologia de etiologia multifatorial, Freire et al. (2014) aponta que as DTMs exigem uma abordagem terapêutica interdisciplinar, mediante uma equipe formada por vários especialistas (cirurgiãodentista, fisioterapeuta, psicólogo e fonoaudiólogo) ou pelo menos uma estreita colaboração entre eles, uma vez que o tratamento bem-sucedido das DTMs deve envolver uma abordagem transdisciplinar e individualizada para cada paciente.

O tratamento fisioterapêutico baseia-se, de uma forma geral, em exercícios, massagens, alongamentos, terapia de liberação posicional, estimulação elétrica nervosa transcutânea, ultrassom e laser. Nos casos em que a DTM está relacionada com alterações posturais, a fisioterapia mostra-se efetiva nos objetivos de evitar a cirurgia, reposicionar a mandíbula, minimizar a dor muscular, melhorar a amplitude de movimento, melhorar a postura, reduzir a inflamação, reduzir a carga na ATM e fortalecer o sistema musculoesquelético (PELICIOLI et al., 2017).

Diante deste contexto, o tema surge em termos profissionais de forma a contribuir, uma vez que a intervenção fisioterapêutica é importante na funcionalidade do indivíduo com Disfunção Temporomandibular. Tendo em vista a importância da temática, este trabalho consiste em uma revisão integrativa da literatura, que tem como objetivo analisar a abordagem fisioterapêutica no tratamento da disfunção temporomandibular, descrevendo os objetivos e resultados das técnicas e recursos utilizados.

\section{METODOLOGIA}

O estudo trata-se de uma revisão integrativa, que é considerada a mais ampla abordagem metodológica dentre as revisões. Este método surgiu a partir da necessidade de assegurar uma prática assistencial embasada em evidências científicas, que é apontada como uma ferramenta ímpar no campo da saúde, pois sintetiza as pesquisas disponíveis sobre determinada temática com 0 intuito de direcionar a prática fundamentando-se em conhecimento científico (SCHLITTLER, 2008).

Para a coleta de dados, foi realizado um levantamento bibliográfico nas bases de dados indexadas na Biblioteca Virtual de Saúde: PubMed, SciELO e LILACS, onde foram utilizados os descritores, descritores em inglês: "Temporomandibular Joint", "Physycal Therapy Specialty" e "Physical Therapy Modalities". Também foi realizada uma busca em língua portuguesa, utilizado os operadores boleanos OR e AND, sem restrição linguística.

A busca foi realizada no período de janeiro a fevereiro de 2018, sendo incluídos artigos publicados entre os anos de 2010 a 2018, na língua portuguesa e inglesa, publicados na íntegra e que abordassem a intervenção fisioterapêutica em pacientes portadores da disfunção temporomandibular. Foram excluídos resumos, textos incompletos, periódicos pagos, relatos e estudos de caso, revisões e trabalhos sem fundamentação científica. 


\section{RESULTADOS}

A partir das associações de descritores "Temporomandibular Joint" AND "Physycal Therapy Specialty", e "Temporomandibular Joint" OR "Physical Therapy Modalities", encontrou-se 169 estudos. Após a filtragem relativa ao ano (2010 a 2018), idioma (português e inglês), texto completo e gratuito disponível, restaram 56 estudos. Estes, por sua vez, tiveram seus títulos e resumos analisados criteriosamente, onde, excluiu-se todos os que não possuíssem abordagem fisioterapêutica na DTM, revisões de literatura, estudos e relatos de caso, restando apenas 7 ensaios clínicos, conforme demonstrado na Figura 1. Em relação aos artigos selecionados, estes estão descritos no Quadro 1.

Figura 1: Etapas metodológicas para seleção dos artigos.

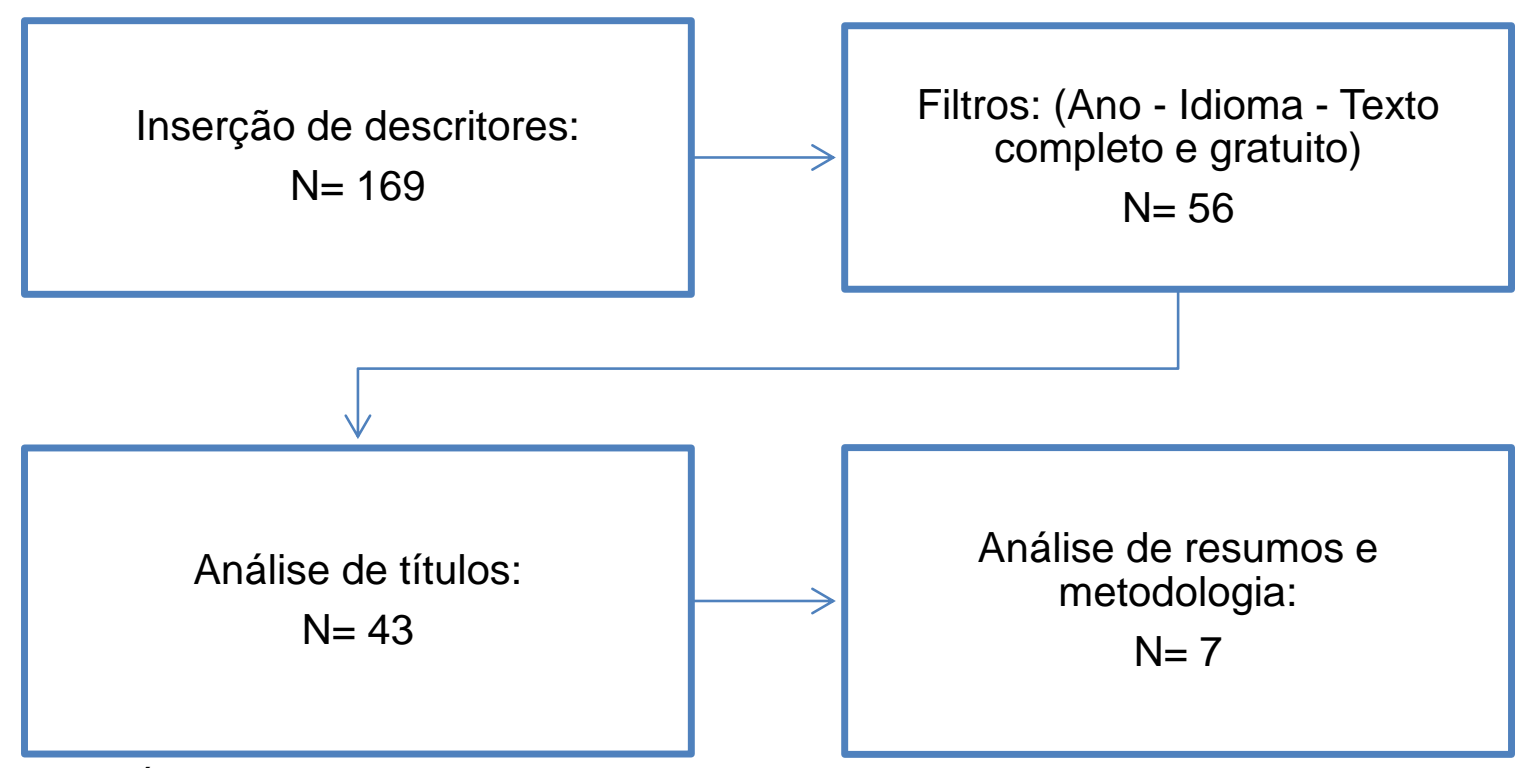

Fonte: PRÓPRIO AUTOR (2018).

\section{DISCUSSÃO}

A disfunção temporomandibular é representada por um grupo de condições que podem ser dolorosas ao paciente e envolvem fatores de predisposição, início e perpetuação dos sintomas, tendo ainda uma relação direta com outras disfunções como o desvio postural da cabeça, coluna cervical e ombros, fazendo com que o indivíduo sinta dor e em casos mais graves, ocorra até o desalinhamento postural do mesmo (AZATO et al., 2013). Nesse âmbito, é valido mencionar que, devido à gravidade e a persistência dos sintomas, estudos que demonstrem a abordagem fisioterapêutica no tratamento da DTM são pertinentes e cada vez mais realizados, e, apesar da carência de profissionais especialistas no tema, é possível observar na literatura vigente grandes indícios dos benefícios da abordagem multidisciplinar da DTM, onde, tratando-se da fisioterapia, observa-se que existem diversos recursos e técnicas disponíveis e eficazes para a melhora do quadro doloroso, e também para a prevenção e tratamento de possíveis alterações secundárias.

Nisto, os estudos analisados utilizaram técnicas e recursos fisioterapêuticos comumente utilizados para o tratamento da DTM, sendo eles a cinesioterapia (uso do movimento passivo ou ativo do paciente para restaurar a função), o tens (impulsos elétricos aplicados na pele para alívio da dor), ultrassom (terapia por ondas eletromagnéticas com o intuito de analgesia, melhora da circulação sanguínea e cicatrização), estimulação elétrica de alta voltagem catótida (estímulos elétricos para analgesia), termoterapia (utilização do calor e do frio como recurso terapêutico para analgesia, redução de edemas e estímulo a circulação sanguínea local) e a acupuntura (aplicação de agulhas em pontos específicos para estímulo das terminações nervosas), onde observa-se que cada recurso/técnica possui uma forma de atuação no sintoma apresentado pelo paciente, possuindo o objetivo principal de restaurar a função do indivíduo. 
Quadro 1: Descrição dos artigos incluídos no trabalho.

\begin{tabular}{|c|c|c|c|c|}
\hline AUTOR / ANO & $\begin{array}{l}\text { TIPO DE } \\
\text { ESTUDO / } \\
\text { AMOSTRA }\end{array}$ & OBJETIVO & $\begin{array}{l}\text { RECURSO - TÉCNICA } \\
\text { FISIOTERAPÊUTICA }\end{array}$ & CONCLUSÃO \\
\hline $\begin{array}{l}\text { Azato et al. } \\
(2013)\end{array}$ & $\begin{array}{l}\text { Ensaio clínico / } \\
30 \text { pacientes. }\end{array}$ & $\begin{array}{l}\text { Avaliar a influência do tratamento da disfunção } \\
\text { temporomandibular muscular na postura global de } \\
\text { indivíduos diagnosticados por meio do Research } \\
\text { Diagnostic Criteria for Temporomandibular } \\
\text { Disorders. }\end{array}$ & $\begin{array}{l}\text { Termoterapia, massagem } \\
\text { local. }\end{array}$ & $\begin{array}{l}\text { O tratamento fisioterapêutico da DTM é } \\
\text { eficaz para a melhora da postura global } \\
\text { por atuar na melhora da dor, reduzindo } \\
\text { adaptações e reorganizações posturais } \\
\text { secundárias ao processo doloroso. }\end{array}$ \\
\hline $\begin{array}{l}\text { Amaral et al. } \\
(2013)\end{array}$ & $\begin{array}{l}\text { Ensaio clínico } \\
\text { controlado / } 50 \\
\text { pacientes. }\end{array}$ & $\begin{array}{l}\text { Avaliar o efeito imediato da mobilização mandibular } \\
\text { não específica (NMM) no controle postural de } \\
\text { indivíduos diagnosticados ou não com DTM. }\end{array}$ & $\begin{array}{l}\text { Mobilização mandibular não } \\
\text { específica. }\end{array}$ & $\begin{array}{l}\text { A mobilização mandibular contribuiu } \\
\text { para a melhoria imediata do controle } \\
\text { postural em pacientes com DTM. }\end{array}$ \\
\hline $\begin{array}{l}\text { Borin et al. } \\
(2011)\end{array}$ & $\begin{array}{l}\text { Ensaio clínico / } \\
40 \text { pacientes. }\end{array}$ & $\begin{array}{l}\text { Avaliar o efeito da acupuntura no nível de dor e } \\
\text { gravidade a disfunção temporomandibular. }\end{array}$ & Acupuntura. & $\begin{array}{l}\text { A acupuntura mostrou-se eficaz na } \\
\text { melhora do nível da dor e da gravidade } \\
\text { da DTM. }\end{array}$ \\
\hline $\begin{array}{l}\text { Ferreira et al. } \\
(2017)\end{array}$ & $\begin{array}{l}\text { Ensaio } \\
\text { randomizado / } \\
40 \text { pacientes. }\end{array}$ & $\begin{array}{l}\text { Investigar o efeito a curto prazo da estimulação } \\
\text { nervosa elétrica transcutânea (TENS), examinando } \\
\text { a intensidade da dor, o limiar de dor de pressão } \\
\text { (PPT) e a atividade de eletromiografia (EMG) em } \\
\text { pacientes com transtorno temporomandibular } \\
\text { (DTM). }\end{array}$ & Tens. & $\begin{array}{l}\text { O tens apresentou efeitos terapêuticos a } \\
\text { curto prazo, diminuindo a dor facial } \\
\text { relatada, e melhorando a percepção de } \\
\text { sensibilidade à dor profunda, revelado } \\
\text { através da melhora da atividade EMG } \\
\text { muscular mastigatória. }\end{array}$ \\
\hline $\begin{array}{l}\text { Gomes et al. } \\
\text { (2011) }\end{array}$ & $\begin{array}{l}\text { Ensaio clínico } \\
\text { randomizado / } \\
20 \text { mulheres. }\end{array}$ & $\begin{array}{l}\text { Avaliar o efeito da estimulação elétrica de alta } \\
\text { voltagem catódica (EEAV) sobre a intensidade da } \\
\text { dor em mulheres com DTM. }\end{array}$ & $\begin{array}{l}\text { Estimulação elétrica de alta } \\
\text { voltagem catódica. }\end{array}$ & $\begin{array}{l}\text { A EEAV mostrou-se eficaz na redução da } \\
\text { intensidade da dor provocada pela DTM. }\end{array}$ \\
\hline $\begin{array}{l}\text { Torres et al. } \\
(2012)\end{array}$ & $\begin{array}{l}\text { Ensaio clínico / } \\
10 \text { pacientes. }\end{array}$ & $\begin{array}{l}\text { Verificar os efeitos dos tratamentos fisioterapêutico } \\
\text { e odontológicos na redução da dor em pacientes } \\
\text { com DTM. }\end{array}$ & 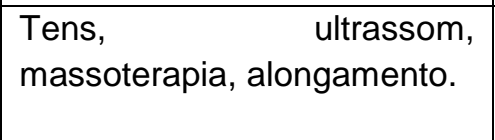 & $\begin{array}{l}\text { A fisioterapia demonstrou ser um } \\
\text { tratamento indicado para o alívio da } \\
\text { sintomatologia dolorosa a curto prazo. }\end{array}$ \\
\hline $\begin{array}{l}\text { Viana et al. } \\
(2016)\end{array}$ & $\begin{array}{l}\text { Ensaio clínico / } \\
60 \text { pacientes. }\end{array}$ & $\begin{array}{l}\text { Avaliar efeitos de um protocolo fisioterapêutico na } \\
\text { qualidade de vida de pacientes com disfunção } \\
\text { temporomandibular. }\end{array}$ & $\begin{array}{l}\text { Ultrassom, mobilização } \\
\text { articular, alongamento. }\end{array}$ & $\begin{array}{l}\text { A aplicação de um protocolo } \\
\text { fisioterapêutico foi capaz de melhorar a } \\
\text { qualidade de vida de pacientes com } \\
\text { disfunção temporomandibular. }\end{array}$ \\
\hline
\end{tabular}

Fonte: PRÓPRIO AUTOR, (2018). 
Nessa linha de pensamento, dentre os recursos que podem ser utilizados, a eletroterapia é apontada como uma grande aliada possuindo grande embasamento científico, onde o tens, conforme demonstrado por Ferreira et al. (2017), apresenta resultados a curto prazo quando comparado com outras terapias, dessa maneira, diminuindo a dor facial relatada, melhorando a sensibilidade do indivíduo e proporcionando um retorno mais rápido às atividades de vida diária do paciente.

Corroborando com tais dados, Torres et al. (2012), complementa que o tens, associado ao ultrassom e a cinesioterapia representada por alongamentos e mobilizações, é eficaz para o alívio da sintomatologia dolorosa, onde, em seu estudo, após dez sessões de atendimento fisioterapêutico com as técnicas referidas, conseguiu observar através da Escala Visual Analógica da Dor e da versão brasileira do Questionário de McGill de Dor, a melhora da percepção da dor pelos pacientes.

Nesse âmbito da eletroterapia, menciona-se ainda o estudo de Gomes et al. (2011), o qual, por sua vez, decidiu avaliar o efeito da estimulação elétrica de alta voltagem catódica (EEAV) na intensidade da dor em pacientes com DTM, e observou que, após dez aplicações de EEAV, houve uma redução da intensidade da dor no grupo intervenção, corroborando com os dados dos autores supracitados de que a eletroterapia pode e deve ser utilizada no tratamento da dor de pacientes com DTM e demonstrando que as terapia relacionadas a estímulos elétricos possuem grande aceitação por parte dos pacientes e eficácia comprovada na literatura abordada.

Ainda tratando-se da atuação fisioterapêutica, esta também é responsável pela mobilidade da ATM, que fica prejudicada na existência de alguma disfunção. Neste âmbito, Amaral et al. (2013) aponta que a mobilização mandibular não específica pode contribuir diretamente para o controle postural de pacientes com DTM, tendo seu pensamento embasado por Azato et al. (2013), que, utiliza a massagem local e a termoterapia como recursos utilizados para o tratamento da dor e das disfunções apresentadas em pacientes com disfunção temporomandibular, os quais abordam em seus estudos ainda que, as alterações posturais causadas pela DTM, se não tratadas, podem contribuir diretamente para a persistência e reincidência dos sintomas, devendo o profissional estar sempre atento as alterações globais apresentadas pelo paciente.

Nisto, Viana et al. (2016) complementa os autores supracitadas abordando que, como forma de tratamento, podem ser utilizadas ainda técnicas simples, como o ultrassom, a mobilização articular e os alongamentos, os quais podem melhorar a qualidade de vida dos pacientes, assim como técnicas mais complexas e menos comuns, como a acupuntura, evidenciada por Borin et al. (2011) por sua eficácia na melhora do nível da dor e da gravidade da DTM.

Concluindo os dados discutidos pelos autores, é válido mencionar ainda que existem outras técnicas não relacionadas somente ao tratamento específico da DTM mas que estão relacionadas aos sintomas globais do paciente que possui determinada disfunção, onde, é pertinente mencionar o uso de terapias que trabalhem a cefaleia tensional e os desarranjos musculares do paciente portador de DTM, as quais tem seu espaço no tratamento multidisciplinar, onde vale a pena finalizar esta discussão mencionando o uso da hidroterapia comprovado por Pinto et al. (2017), que é eficaz na redução das tensões musculares e alívio da dor, bem como também pode-se corroborar com as terapias alternativas o uso de técnicas de alinhamento postural demonstradas por Chaves et al. (2017) para o alívio das cadeias musculares e restauração da postura e funcionalidade comprovada em 117 pacientes que sofriam com a DTM. Dessa maneira, demonstra-se que a DTM possui e merece não somente os sintomas específicos e tratamentos isolados da articulação afetada, mas ainda uma abordagem global do paciente.

\section{CONSIDERAÇÕES FINAIS}

A partir dos resultados deste estudo pode-se concluir que a disfunção temporomandibular pode causar limitações e diminuição da qualidade de vida de seus pacientes por conta do quadro álgico doloroso comumente referido como sintoma. Nisto, a fisioterapia, associada ao tratamento de uma equipe multidisciplinar, pode proporcionar um alívio mais rápido da dor sentida e referida pelos portadores de DTM, contribuindo, dessa forma, para uma melhor qualidade de vida dos mesmos.

Dessa forma, a abordagem fisioterapêutica no tratamento da DTM tem o objetivo de reduzir o quadro álgico, e melhorar a mobilidade da ATM, diminuindo possíveis sintomas secundários como a vertigem, contraturas, dificuldade de mastigação ou deglutição. Para tanto, recursos elétricos como o tens e o ultrassom, recursos térmicos como o gelo e o calor, e recursos manuais como alongamentos, massagens e mobilizações 
são amplamente utilizados e comentados na literatura vigente, por, comprovarem sua eficácia no tratamento das disfunções temporomandibulares.

\section{REFERÊNCIAS}

1. AMARAL AP, POLITTI F, HAGEL YE et al. Immediate effect of nonspecific mandibular mobilization on postural control in subjects with temporomandibular disorder: a single-blind, randomized, controlled clinical trial. Braz $\mathrm{J}$ Phys Ther., v. 17, n. 2, p. 121-127, 2013.

2. AZATO FK, CASTILLO DB, COLHO TMK et al. Influence of temporomandibular disorders management on pain and global posture. Rev Dor, v. 14, n. 4, p. 280-283, 2013.

3. BONOTTO D, CUSTÓDIO LG, CUNALI PA. Viscossuplementação como tratamento das alterações internas da articulação temporomandibular - Relato de casos. Rev Dor, v. 12, n. 3, p. 274-278, 2011.

4. BONOTTO D, MACHADO E, CUNALI RS et al. Viscosupplementation as a treatment of internal derangements of the temporomandibular joint: retrospective study*. Rev Dor, v. 15, n. 1, p. 2-5, 2014.

5. BORIN GS, CORRÊA EC, SILVA AMT et al. Acupuntura como recurso terapêutico na dor e na gravidade da desordem temporomandibular. Fisioter Pesq., v. 18, n. 3, p. 217-222, 2011.

6. CHAVES PJ, OLIVEIRA FEM, DAMÁZIO LCM. Incidence of postural changes and temporomandibular disorders in students. Acta Ortop Bras, v. 25, n. 4, p. 162-164, 2017.

7. FERREIRA APL, COSTA DRA, OLIVEIRA AIS et al. Short-term transcutaneous electrical nerve stimulation reduces pain and improves the masticatory muscle activity in temporomandibular disorder patients: a randomized controlled trial. J Appl Oral Sci., v. 25, n. 2, p. 112-120, 2017.

8. FREIRE AB, NARDI AT, BOUFLEUR $\mathrm{J}$ et al. Multimodal physiotherapeutic approach: effects on the temporomandibular disorder diagnosis and severity. Fisioter Mov., v. 27, n. 2, p. 219-227, 2014.

9. GOMES NCMC, SCHWARZENBECK KCSB, PACKER AC et al. Efeito da estimulação elétrica de alta voltagem catódica sobre a dor em mulheres com DTM. Rev Bras Fisioter., v. 16, n. 1, p. 10-15, 2012.

10. MILANESI JM, WEBER P, PASINATO $F$ et al. Severidade da desordem temporomandibular e sua relação com medidas cefalométricas craniocervicais. Fisioter Mov., v. 26, n. 1, p. 79-86, 2013.

11. PELICIOLI M, MYRA RS, FLORIANOVICZ VC et al. Physiotherapeutic treatment in temporomandibular disorders. Rev Dor, v. 18, n. 4, p. 355-361, 2017.

12. PINTO DR, MORAIS J, FERREIRA ALN et al. Abordagem não-farmacológica na cefaleia do tipo tensional: efeitos da hidroterapia sobre a dor e a qualidade de vida. Rev Bras Neurol., v. 53, n. 1, p. 15-26, 2017.

13. SCHLITTLER JM. Como Fazer Monografias. Campinas: Servanda, 2008.

14. TORRES F, CAMPOS LG, FILLIPINI HF et al. Efeitos dos tratamentos fisioterapêutico e odontológico em pacientes com disfunção temporomandibular. Fisioter Mov., v. 25, n. 1, p. 117-125, 2012.

15. VIANA MO, OLEGARIO NBC, VIANA MO et al. Effect of a physical therapy protocol on the health related quality of life of patients with temporomandibular disorder. Fisioter Mov., v. 29, n. 3, p. 507-514, 2016.

15. WEBER P, CORRÊA ECR, BOLZAN GP et al. Mastigação e deglutição em mulheres jovens com desordem temporomandibular. CoDAS, v. 25, n. 4, p. 375-380, 2013. 